\title{
Space Time Trellis Code Frequency Index Modulation with Neuro-LS Channel Estimation in OFDM
}

\author{
Sarita Yadav \\ M.Tech. Scholar \\ Department of EC \\ PCST \\ Bhopal, M.P, India \\ yadavsarita622@gmail.com
}

\author{
Ashish Nema \\ Assistant Professor \\ Department of EC \\ PCST \\ Bhopal, M.P, India
}

\author{
Jitendra Mishra \\ Associate Professor \\ Department of EC \\ PCST \\ Bhopal, M.P, India
}

\begin{abstract}
In wireless communication, orthogonal frequency division multiplexing (OFDM) plays a major role because of its high transmission rate. In space-time shift keying (STSK), the information is conveyed by both the spatial and time dimensions, which can be used to strike a trade-off between the diversity and multiplexing gains. On the other hand, orthogonal frequency division multiplexing (OFDM) relying on index modulation (IM) conveys information not only by the conventional signal constellations as in classical OFDM, but also by the indices of the subcarriers. In this paper compressed sensing(CS) is studied in order to increase throughput and bit-error performance by transmitting extra information bits in each subcarrier block as well as to decrease the complexity of the detector. In this paper, soft trellis decoding algorithm is implemented with channel estimation using Neuro-LS technique. The result analysis shows the better performance of trellis decoder with respect to BER and Neuro-LS channel estimation with respect to BER.
\end{abstract}

Keywords: OFDM, MIMO, Space Time Trellis Code, Frequency Index Modulation, Compressed Sensing (CS), Channel Estimation

\section{INTRODUCTION}

OFDM is a wideband wireless digital communication technique that is based on block modulation. With the wireless multimedia applications becoming more and more popular, the required bit rates are achieved due to OFDM multicarrier transmissions. Multicarrier modulation is commonly employed to combat channel distortion and improve the spectral efficiency. Multicarrier Modulation schemes divide the input data into bands upon which modulation is performed and multiplexed into the channel at different carrier frequencies so that information is transmitted on each of the sub carriers, such that the sub channels are nearly distortion less [1].

Space-time shift keying (STSK) has been regarded as an advantageous technique because of its flexibility in providing both multiplexing and transmit diversity gains [2]. Compared to the conventional spatial modulation (SM) [3] and space-shift keying (SSK) [4], STSK exhibits both transmit and receive diversity gains instead of only attaining receive diverity gain, as in SM and SSK.

Namely, STSK spreads information to both the spatial and time dimensions, where the information is mapped to the classic L-PSK/QAM symbols and additional information is transmitted by activating one out of Q dispersion matrices (DM). On the other hand, the distortion due to multipath fading is the main challenge in wideband fading channels, and often MultiCarrier (MC) modulation is used for mitigating this distortion, which effectively converts the dispersive wideband channels into a number of parallel narrowband flat-fading subchannels [5].

Orthogonal frequency-division multiplexing (OFDM) is the most widespread MC modulation technique, as a benefit of its robustness in wideband channels and its low-complexity implementation. OFDM has been combined with STSK.

Index modulation (IM) [6] is an emerging concept, in which extra information bits are mapped to the indices of multiple transmission resources, such as the indices of antennas, subcarriers or time slots. By contrast, OFDM with IM (OFDM-IM) is a beneficial frequency-domain IM technique, which has been proposed as an alternative to classical OFDM. In OFDM-IM not all available subcarriers are activated. For example, activating one out of four subcarriers allows us to convey two extra information bits per subcarrier block.

Ishikawa et al. [12] show that OFDM-IM improves the error performance at low values of signal-to-noise ratio (SNR) compared 
to the classical OFDM over frequency-selective fading channels. In order to further improve the spectral efficiency of OFDM modulated systems, a variable number of active subcarriers per OFDM subblock was applied in [17], where the frequencydomain IM is generalized and the problem of selecting the optimal number of active subcarriers for OFDMIM is investigated.

IM concept can be considered for other communications systems apart from MIMO systems. For an instance, IM techniques can be efficiently implemented for the subcarriers of an OFDM system. OFDM-IM is a novel multi-carrier transmission scheme which has been proposed by inspiring from the IM concept of SM [21].

Similar to SM, in the OFDM-IM scheme, the incoming bit stream is split into index selection and $\mathrm{M}$ ary constellation bits. According to the index selection bits, only a subset of available subcarriers are selected as active, while the remaining inactive subcarriers are not used and set to zero. On the other hand, the active subcarriers are modulated according to the M-ary constellation bits (as shown in figure 1).

In other words, the information is conveyed not only by the data symbols as in classical OFDM, but also by the indices of the active subcarriers which are used for the transmission of the corresponding data symbols for the OFDM-IM scheme.

\section{METHODOLOGY}

The block diagram of the proposed scheme is shown in Fig. 1, where $b_{N}$ number of information bits are divided into $\mathrm{N}$ parallel groups with $\mathrm{b}$ number of information bits processed in each group, as shown in Fig. 1. For each group of $b$ bits, $b_{1}$ bits are mapped to the IM selector, which chooses $\mathrm{K}$ active indices out of $\mathrm{N}_{\mathrm{a}}$ available indices. The remaining $b_{2}$ bits are used for generating K STTC codewords, and then these K codewords are coordinate-interleaved for providing an additional diversity gain to improve the BER and MSE performance. The K coordinate interleaved codewords are then mapped to the active indices according to the IM selector, while the inactive indices are set to zero. Then the block creator collects all codewords from $\mathrm{G}$ groups in parallel and forms a frame, which is mapped to the space-time trellis codewords followed by DWTOFDM modulation and then transmission. 


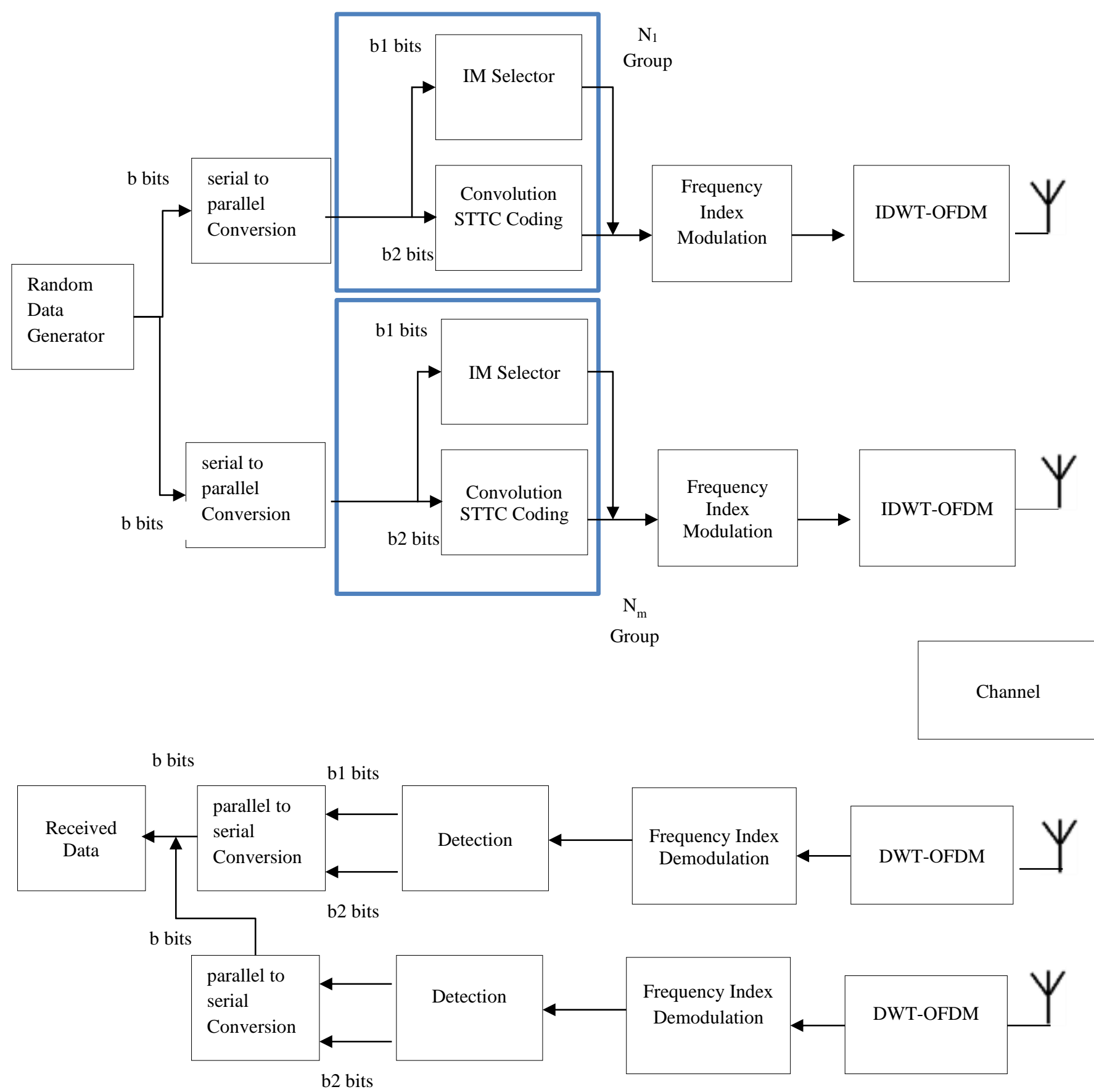

Fig. 1. The transceiver architecture

In the system proposed in Fig. 1, we consider OFDM modulation with Nc subcarriers, which are equally divided into $\mathrm{N}$ subcarrier groups and each group contains $\mathrm{Mg}=\mathrm{Nc} / \mathrm{N}$ subcarriers in the frequency domain. In each subcarrier group, $\mathrm{K}$ number of indices are active out of $\mathrm{Na}$ available subcarrier indices in the virtual domain. In OFDM, Nc may assume very large values, and if the index selector is applied directly to $\mathrm{Nc}$, there could be a huge number of possible combinations for active indices, which makes the selection of active indices an almost impossible task. As a result, the subcarriers are partitioned into $\mathrm{N}$ smaller groups to perform index selection. As shown in Fig. 1, the information bits are divided into G groups at the input of the transmitter. The details of the transmitter and receiver models are discussed in the following sections.

\subsection{Transmitter Model}

As illustrated in Fig. 1, bG data bits are split into $\mathrm{N}$ groups of length $\mathrm{b}$ bits and then the corresponding $\mathrm{b}$ bits are processed in each group of the transmitter by the convolution STTC encoder and the IM selector. The convolution codes are different from the square 
codes in the operational strategy method. A convolutional encoder works on serial information, although the part codes work on a square of information. The use of memory components in convolutional encoders is also unique. In the period of encrypted information, no memory component is required due to part codes.

The convolutional codes are indicated in the form (n, $\mathrm{k}, \mathrm{L}$ ), where $\mathrm{n}$ is the set of efficiency bits of the encoder, $\mathrm{k}$ is the set of information bits of the encoder and $\mathrm{L}$ is the imperative length of this encoder. The length of the request is used to calculate the amount of memory or flip-lemon used in the encoder. Whatever time period we know $\mathrm{L}$ and the fundamental equation, we can calculate all memory levels (m).

$$
\mathrm{L}=\mathrm{k} \times(\mathrm{m})
$$

We will take up a straightforward convolution code $(2,1,3)$ where $\mathrm{n}=2, \mathrm{k}=1$ and $\mathrm{L}=3$ ( the expression $\mathrm{L}=\mathrm{k}(\mathrm{m}+1)$ is used).Lets build the encoder from the above data. The encoder is developed with 1 input bit, 2 yield bits and 2 memory components. Take note of that the $\mathrm{L}=\mathrm{k}(\mathrm{m}+1)$ expression prompts to 2 memory components. Here these two memory components are utilized to store the previous 2 input bits. On the off chance that the expression $\mathrm{L}=\mathrm{k} * \mathrm{~m}$ is utilized and for a $(2,1,3)$ encoder $(\mathrm{L}=3)$, the quantity of memory components will be 3 , where these 3 memory components are utilized to store past 3 input bits. So the expression for imperative length must be deliberately translated, generally any distortion will prompt to an alternate encoder structure out and out [23].

Presently we know the quantity of bits going into the encoder, number of bits turning out from it and the quantity of memory components. Till now the encoder resembles a black box to us as in we don't know how the memory components are used to create the yield bits from the information. To completely comprehend the encoder structure we require something many refer to as "generator polynomials" that let us know how the memory components are connected to accomplish encoding. The generator polynomials for a particular convolutional encoder set $(n, k, L)$ are generally found through recreation. The set $(\mathrm{n}, \mathrm{k}, \mathrm{L})$ alongside $\mathrm{n}$ generator polynomials totally depicts a convolutional encoder.

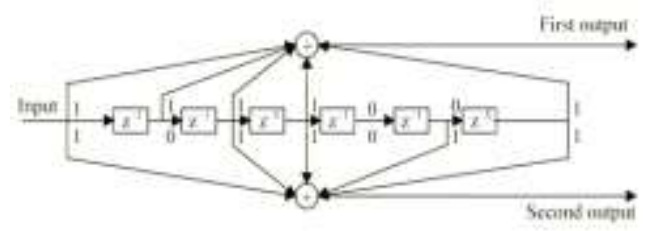

Fig. 2. Convolutional Encoder Structure
The convolutional encoder can also be represented using a finite state machine. The entire behavior of a convolutional encoder is depicted by a state diagram. The number of states in a state diagram depends on the number of memory elements in the encoder. If number of memory elements is $m$ then the number of states in the state diagram will be $2 \mathrm{~m}$. Poly2trellis is used here for encoding. 133 and 171 are in octal which are 1011011 and 1111001 in binary. Puncturing is also added to make rate $3 / 4$. Puncturing is a periodic omission of encoded bits. The pattern used is 111001 . This means for 3 bit input, 6 bits are produced of which 4 th and 5 th bit are omitted.

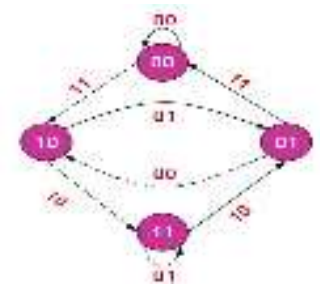

Fig. 3. State Diagram of Convolutional Encoder

The four conceivable conditions of the encoder are delineated as four even lines. Exhibit states are thought to be on the left side and the conceivable next states on the correct side of the trellis. The associations between the present states and the following states precisely take after the state chart representation. Once more, the yield bits produced amid every state move are demonstrated in dull red shading. A different stage Trellis is developed by recreating the single stage and comparing each of the single stage one next to the other as appeared in the figure 3.9.

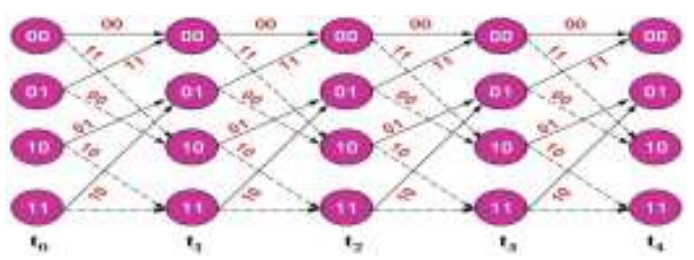

Fig. 4. Trellis diagram of four stages

As illustrated in Fig.1, the OFDM symbols in the frequency domain are obtained after the above procedures, and then the Nc-point inverse discrete wavelet transform (IDWT) is applied at each transmit antenna. Let $\mathrm{X}_{\mathrm{N}}$ be the signal that is to be transmitted over $\mathrm{M}_{\mathrm{t}}$ and $\mathrm{M}_{\mathrm{r}}$ antennas. The signal $\mathrm{X}_{\mathrm{N}}$ is the group of $\mathrm{X}_{\mathrm{N} 1} . . \mathrm{X}_{\mathrm{Nm}}$ and $\mathrm{XN}_{1}$ can be expressed as $\mathrm{x}=\left[\mathrm{b}_{1}(\mathrm{~m})\right.$ and $b_{2}(m)$ ] for $m_{\text {th }}$ subcarrier and for $i_{\text {th }}$ transmitting antenna $x_{i}=\left[x_{i}(1), x_{i}(2), \ldots \ldots ..\right]$. After IDWT is applied using equation that is represented as:

$$
d(k)=\sum_{m=0}^{\infty} D_{m}^{n} 2^{\frac{m}{2}} \psi\left(2_{k}^{m}-n\right)
$$


Where $\mathrm{D}_{\mathrm{m}}^{\mathrm{n}}$ are the wavelet coefficients and $\psi(\mathrm{t})$ is the wavelet function with compressed factor $m$ times and shifted $\mathrm{n}$ times for each subcarrier (number $\mathrm{k}, 0 \leq \mathrm{k} \leq$ $\mathrm{N}-1$ ). The wavelet coefficients are the representation of signals in scale and position or time. At the receiver side, the process is inversed.

Then signal is transmitted as shown in fig 5 .

$$
x^{\prime}=x+n
$$

Where, $\mathrm{x}$ is transmitting signal and $\mathrm{n}$ is noise added in the channel. The new signal is modified with noise. So, the receiving $M_{r}$ will receive $x^{\prime}$.

Finally, the cyclic prefix (CP) of length Lcp, which has to be longer than the channel's delay spread, is concatenated at the front of the symbols for eliminating the effects of the intersymbol-interference over multipath fading channels. Then, the resultant signals are transmitted simultaneously from the $\mathrm{M}$ transmit antennas over the wireless channel.

\subsection{Receiver Model}

Using the channel model, at the $n_{c}$ th subcarrier between the m-th transmit antenna as well as the n-th receive antenna and following the $\mathrm{CP}$ removal and DWT operation.

$$
\mathrm{Y}[\mathrm{n}]=\mathrm{H}[\mathrm{n}] * \mathrm{~S}[\mathrm{n}]+\text { noise }[\mathrm{n}]
$$

Where, $Y[n]=$ Received at $n_{\text {th }}$ sub-carrier.

$\mathrm{H}[\mathrm{n}]=$ Channel Matrix

$\mathrm{S}[\mathrm{n}]=$ Compressed $\mathrm{b}_{\mathrm{g}}$ transmitted bits

Noise $[\mathrm{n}]=$ noise in the channel

The received signal $\mathrm{Y}[\mathrm{n}]$ is then passed through the block splitter and the received symbols in $\mathrm{N}$ groups are detected separately as shown in Fig. 1. Then the received signal $\mathrm{Y}[\mathrm{n}]$ in the $\mathrm{n}$-th group before detection can be obtained. As orthogonal frequency division multiplexing (OFDM) supports a high data rate in time delay spread environments with efficient equalization. As equalization requires Channel State Information (CSI), pilots on predetermined subcarriers are sent as training signals for OFDM systems. In the channel estimation, it is assumed that intersymbol interference is dropped by guard interval, and the received signal is considered as in equation :

$\mathrm{Y}(\mathrm{k})=\mathrm{X}(\mathrm{k}) \mathrm{H}(\mathrm{k})+\mathrm{W}(\mathrm{k}) \quad$ Where $\mathrm{Y}(\mathrm{k})$ is the received signal vector, $\mathrm{X}(\mathrm{k})$ is a matrix containing the transmitted signaling points on its diagonal, $\mathrm{H}(\mathrm{k})$ is a channel attenuation vector, and $\mathrm{W}(\mathrm{k})$ is a noise vector.

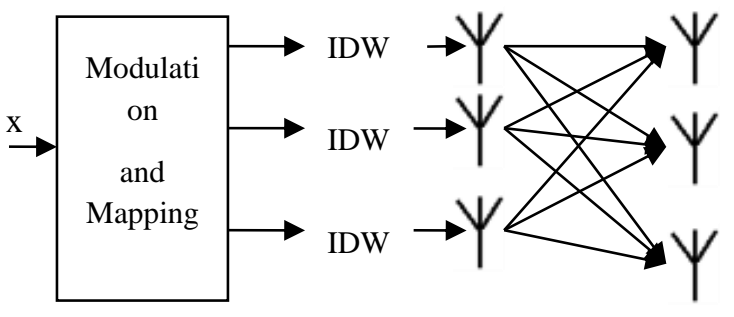

\section{Fig 5. IDWT based OFDM Transmitter and Receiver Block}

The data is transmitted in noisy channel. The channel is Rayleigh fading with AWGN noise. The equation for this transmission is given below:

A Neuro-LS Estimator has designed for channel estimation and equalization. In Neuro-LS channel estimation method back propagation neural network is designed. Backpropagation is a common method for training a neural network. For training process, bayesian regularization is used for minimizing a linear combination of squared errors and weights. For this proposed model a neural network based estimator is designed for channel estimation and equalization. Neuro-Estimator architecture have three layer include input layer, hidden layer and output layer. The received noisy data is sent to the input layer of the neural network and this data is feed forwarded to hidden layer and output of the hidden layer is computed as in equation (xi):

$$
n e t_{j}^{h}=\sum I_{i} W_{i j}, j=1,2, \ldots \ldots . n
$$

where $I_{i}$ is input data of ith units, $w_{i j}$ is input-to-hidden layer weights. Then hidden layer output is obtained by activation function. This output of the hidden layer is forwarded to the final output layer of the network. In output layer, the final neural network output is computed as:

$$
n e t_{o}^{k}=\sum o_{j}^{h} w_{j k}
$$

where $O_{j}^{h}$ is the input to the output layer coming from hidden layer and $\mathrm{w}_{\mathrm{jk}}$ is hidden-to-output layer weights. However, Output layer output expressed as:

$$
O_{k}^{o}=f\left(\text { net }_{k}^{o}\right)
$$

where $O_{k}^{o}$ is output layer output of kth units. In training process, the back propagation learning algorithm is based on Bayesian training function. Bayesian training function minimizes a linear combination of squared errors and weights. It also modifies the linear combination so that at the end of training the network has good generalization qualities. The Neuro-LS channel estimation operation can be divided into the number of steps that represented as follow: 
step 1. Set learning rate and tolerable error.

step 2. The input-to-hidden layer weights $\mathrm{w}_{\mathrm{ij}}$ and hidden to output layer weights $\mathrm{w}_{\mathrm{jk}}$ are initialized with random values.

step 3. The training data from the input layer into the network in a certain order.

step 4. Calculated hidden layer output $O_{j}^{h}$ and output layer output $O_{k}^{o}$.

step 5. Calculated error function values $\mathrm{E}$.

step 6. Calculated the weights correction $\Delta \mathrm{w}$.

step 7. Adjusted input-to-hidden layer weights $\mathrm{w}_{\mathrm{ij}}$ and hidden-to-output layer weights $\mathrm{w}_{\mathrm{jk}}$.

step 8. Back to step 3, iterated calculated and correction, until the error between network output and desired output less tolerable error, or achieved maximum training times.

Further, a hard choice viterbi decoder has been utilized to translate the encoded equalized information. The Viterbi Algorithm (VA) finds a most maximum likelihood (ML) estimate of a transmitted code arrangement $\mathrm{c}$ from the comparing got grouping $\mathrm{r}$ by augmenting the likelihood $\mathrm{p}(\mathrm{r} \mid \mathrm{c})$ that succession $\mathrm{r}$ is gotten adapted on the evaluated code arrangement c. Grouping c must be a valid coded arrangement. The basic operations which are carried out as per the harddecision Viterbi Algorithm after receiving one codeword are summarized below:

1. All the branch metrics of all the states are determined;

2. Accumulated metrics of all the paths (two in our example code) leading to a state are calculated taking into consideration the 'accumulated path metrics' of the states from where the most recent branches emerged;

3. Only one of the paths, entering into a state, which has minimum 'accumulated path metric' is chosen as the 'survivor path' for the state (or, equivalently 'node');

4. So, at the end of this process, each state has one 'survivor path'. The 'history' of a survivor path is also maintained by the node appropriately ( e.g. by storing the codewords or the information bits which are associated with the branches making the path);

5. Steps a) to d) are repeated and decoding decision is delayed till sufficient number of codewords has been received.

\section{RESULT ANALYSIS}

In simulation of proposed methodology, Mean Square Error performance is analysed under AWGN channel using Matlab software with frequency index modulation technique. The proposed methodology are simulated and compared with each other with variable signal to noise ratio $(\mathrm{Eb} / \mathrm{No})$ which are shown below. The performance parameters used in this methodology are discussed as below:

Mean Square Error (MSE): It measures the average of the squares of the errors, that is, the average squared difference between the actual values and estimated values.

Bit Error Rate (BER): A bit error rate is defined as the rate at which errors occur in a transmission system. This can be directly translated into the number of errors that occur in a string of a stated number of bits. It is average rate of erroneously decoding the transmitted information bits at the communication receiver.

The comparisons between the classical OFDM-STSK, conventional OFDMSTSK-IM, and the CS-aided OFDM-STSK-IM are presented at the same transmission data rates. The BER performances of these schemes are evaluated by Monte Carlo simulations. Table I shows the system parameters for proposed methodology.

Table I: System Parameters in Simulation

\begin{tabular}{|c|c|}
\hline Parameters & Values \\
\hline Multi-carrier System & OFDM \\
\hline Number of subcarriers & 128 \\
\hline Length of Cyclic Prefix & 16 \\
\hline $\begin{array}{c}\text { Number of subcarrier } \\
\text { groups, N }\end{array}$ & 16 \\
\hline $\begin{array}{c}\text { Number of available } \\
\text { indices/group }\end{array}$ & AWGN/Rayleigh \\
\hline $\begin{array}{c}\text { Number of active } \\
\text { indices/group }\end{array}$ & Fading \\
\hline $\begin{array}{c}\text { Channel Specification } \\
\text { SNR }\end{array}$ \\
\hline
\end{tabular}

Fig. 6. BER Performance of Soft Trellis Decoder Vs SNR

Fig. 6 that the performance of proposed methodology is about $0: 10 \mathrm{~dB}$ and $1 \mathrm{~dB}$ lower than that of ML detector, respectively. More quantitively, the proposed 
algorithm have $10^{-5}$ BER with respect to ML detector. It has been concluded that proposed methodology is sufficient to attain a better performance, despite having a lower decoding complexity. Additionally, in order to further reduce the complexity, Neuro-LS channel estimation and equalization is also performed and it is shown that Neuro-LS have less MSE as compared to traditional LS channel estimation technique. Figure 7 illustrates the performance of the MSE.

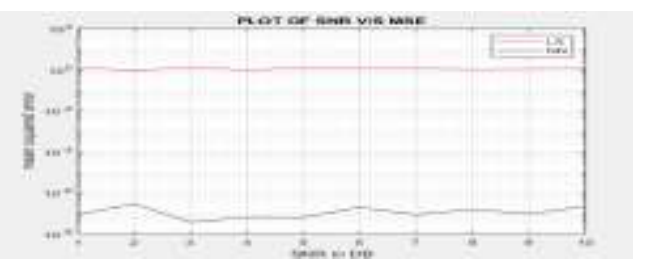

Fig. 7. MSE Performance of Neuro-LS Equalizer with Soft Trellis Decoder Vs SNR

\section{CONCLUSION}

In this paper, a space time trellis code frequency index modulation scheme relying on CS-aided reduced complexity detections for transmission over frequency selective channels. The information bits are transmitted using space, time and frequency dimensions to improve the spectral efficiency as well as the BER performance. In the simulation, the proposed methodology employed soft trellis detector that has the best BER performance than the classical OFDM-STSK system. The Neuro-LS channel estimation gives best MSE performance that traditional MMSE channel estimation techniques.

\section{REFERENCES}

[1] Branka Vucetic and Jinhong Yuan, "Space-Time Coding", John Wiley \& Sons, British Library Cataloguing in Publication Data, (2003).

[2] Dipl.-Ing. Biljana Badic, "Space-Time Block Coding for Multiple Antenna Systems", Dr. Thesis, 2005.

[3] K. Kumar and A. Mitra, "Estimation of MIMO Channels Using Complex Time Delay Fully Recurrent Neural Network", IEEE, 2nd National Conference Emerging Trends and Application in Computer Science (NCETACS), pp. 1-5, 2011.

[4] Kaleeswaran Rajeswari, S. Jayaraman Thiruvengadam, "Optimal Power Allocation for Channel Estimation in MIMO-OFDM System with Per-Subcarrier Transmit Antenna Selection", RADIOENGINEERING, Vol. 24, No. 1, 2015.

[5] Sven Jacobsson_y, "One-Bit Massive MIMO: Channel Estimation and High-Order Modulations", Chalmers University of Technology, 2015.

[6] Z. Ling and Z. Xianda, "MIMO Channel Estimation and Equalization using Three-Layer Neural Networks with Feedback", IEEE, Vol. 12, No.6, pp. 658- 662, 2007.

[7] C. Çiflikli, A. Tuncay Özsahin and A. Çagri, "Artificial Neural Network Channel Estimation Based on LevenbergMarquardt for OFDM Systems", Springer, Science+Business Media, Vol. 51, pp. 221-229, 2008.

[8] K. Charly Jomon and S. Prasanth, "Artificial Neural NetworkbChannel Estimation Based on Levenberg-
Marquardt for OFDM Systems", ISSN 0735-2727, Radio electronics and Communications Systems, Vol. 60, No. 2, pp 80-87. (C) Allerton Press, Inc., 2017.

[9] Y. Chau and S.-H. Yu, "Space modulation on wireless fading channels," Proc. IEEE VTC'2001, Vol. 3, pp. 1668-1671, October 2001.

[10] I. A. Hemadeh, M. El-Hajjar, S. Won, and L. Hanzo, "Layered multigroup steered space-time shift-keying for millimeter-wave communications," IEEE Access, Vol. 4, pp. 3708-3718, April 2016.

[11] E. Basar, "Index modulation techniques for $5 \mathrm{G}$ wireless networks," IEEE Communications Magazine, Vol. 54, No. 7 , pp. 168-175, July 2016.

[12] N. Ishikawa, S. Sugiura, and L. Hanzo, "Subcarrier-index modulation aided OFDM - will it work?" IEEE Access, Vol. 4, pp. 2580-2593, May 2016.

[13] T. Datta, H. S. Eshwaraiah, and A. Chockalingam, "Generalized spaceand-frequency index modulation," IEEE Transactions on Vehicular Technology, Vol. 65, No. 7, pp. 4911-4924, July 2016.

[14] E. Basar, U. Aygolu, E. Panayirci, and H. V. Poor, "Orthogonal frequency division multiplexing with index modulation," IEEE Transactions on Signal Processing, Vol 61, No. 22, pp. 5536-5549, Nov 2013.

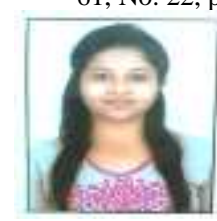

Sarita Yadav, BE in EC from patel college of science and technology RGPV Bhopal, MTECH in digital communications from pate collage of science and technology RGPV Bhopal.

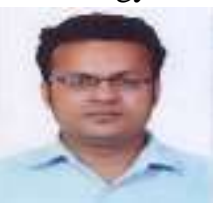

Ashish Nema is working as Assistant Professor, Department of Electronics and communication in PCST, Bhopal (RGPV). He has received Master of Technology DC from RGPV Bhopal and Bachelor of Engineering in EC from RGPV Bhopal. His area of interest is in Optical Communication, Digital Signal Processing, Wireless Communication, Image Processing etc.

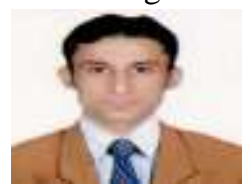

Mr. Jitendra Kumar Mishra is working as Associate Professor and Head of the Department of Electronics and communication in PCST, Bhopal (RGPV). He has received Master of Technology and Bachelor of engineering respectively in Digital communication from BUIT, Bhopal and from RGPV, Bhopal. He has more than 10 years of teaching experience and publish $20+$ papers in International journals, conferences etc. His area of Interests are Antenna \& Wave Propagation, Digital Signal Processing, Wireless Communication, Image $=$ Processing etc . 